\title{
Comparative study between Dexmedetomidine-Ketamine and Propofol-Ketamine Combinations for Anesthesia in Spontaneously Breathing Pediatric Patients Undergoing Cardiac Catheterization
}

\author{
Hassan Ahmed M., Salah Ahmed M., Wesam Abd-elgalil A., Wael Alham M.
}

\section{Abstract \\ Introduction:}

Sedation for pediatric cardiac catheterization is a common requirement in many institutions. The goals of the anesthetic management during cardiac catheterization are adequate analgesia, sedation, immobility, and cardiovascular stability(Cravero et al, 2006).

Ketamine has potential advantages includeexcellent sedation and analgesia and maintenance of airwayreflexes and respiratory drive. Also, it preserves cardiac function(Williams et al, 2007).

Propofol has a predictable onset of action, a short half-life with a rapid recovery time, and is easily titratable. However, propofol is associated with profound respiratory depression, and no analgesic effect(Ewen et al, 1995).

Dexmedetomidine is a highly selevtive $\alpha 2$ adrenoceptor agonist with more specificity for the receptor compared to clonidine, approved by FDA in 1999. It provides excellent sedation and analgesia with minimal respiratory depression (Bhana et al, 2000).

Aim of work:

This study aimed to compare between dexmedetomidine-ketamine and propofol-ketamine combinations in pediatric patients undergoing cardiac catheterization.

\section{Patients and methods:}

After local ethical committee approval an obtained consent from the parents, 44 acyanotic pediatric patients, aged 4 months to 16 years with ASA II and III, analyzed with the chisquare test.

KEY WORDS: Pediatric anesthesia, Cardiac catheterization, Dexmedetomidine, Ketamine, Propofol.

\section{Introduction}

Patients with congenital heart lesionsare a common group of children undergoing pediatric cardiac catheterization (Hickey et al, 1992).

Cardiac catheterization has long served as the "gold standard" for the anatomic and physiological assessment of patients with CHD. It is a procedure that uses long, thin, hollow tubes called catheters to make $\mathrm{x}$-ray pictures of your heart and its blood vessels. The procedure also determines how well the heart muscle and its valves are performing. (Mullins et al, 2006).

Sedation for pediatric cardiac catheterization is a common emergencedelirium (Berman et al, 1990 ; Williams et al, 2007).

Propofol has a predictable onset of action, a short half-life with a rapid requirement in many institutions. The goals of the anesthetic management during cardiac catheterization are adequate analgesia, sedation, immobility, and cardiovascular stability(Cravero et al, 2006).

Ketamine haspotential advantages includeexcellent sedation and analgesia and maintenance of airwayreflexes and respiratory drive. Also, ketamine preserves cardiac function through increasing sympathetic effects.Despite these benefits, there areseveral potefntial problems with ketamine use in children; it isassociated with a prolonged recovery period and recovery time, and is easily titratable. However, propofol is associated with profound respiratory depression, and no analgesic effect (Ewen et al, 1995). 
Ketamine and propofol have opposing influences on blood pressure, heart rate, and SVR. In addition, ketamine supplementing a propofol infusion has been shown to preserve respiratory function and upper airway control (Akin et al, 2005).

Dexmedetomidine is a highly selevtive $\alpha 2$ adrenoceptor agonist with more specificity for the receptor compared to clonidine, approved by FDA in 1999. It provides excellent sedation and analgesia with minimal respiratory depression (Bhana et al, 2000).

\section{Patients and methods:}

After local ethical committee approval an obtained consent from the parents, 44 acyanotic pediatric patients, aged 4 months to 16 years with ASA II and III, who were scheduled for elective cardiac catheterization for evaluation and intervention of congenital heart disease (CHD).

\section{Exclusion criteria}

- Patients with cyanotic CHD.

- Patients required mechanical ventilation.

- Patients required intravenous inotropic support.

- known hypersensitivity to used drugs of sedation informed by the parients.

- Patients refused the study protocol.

The patients are randomly allocated to two equal groups 22 patients each:

Group (1): patients received $1 \mathrm{ug} / \mathrm{kg}$ of dexmedetomidine and $1 \mathrm{mg} / \mathrm{kg}$ of ketamine, as infusion over minutes, for induction. Then,the patients received an infusion of $0.7 \mathrm{ug} / \mathrm{kg} / \mathrm{h}$ dexmedetomidine and $1 \mathrm{mg} / \mathrm{kg} / \mathrm{h}$ ketamine for maintenance.

Group (2):patients received $1 \mathrm{mg} / \mathrm{kg}$ of propofol and $1 \mathrm{mg} / \mathrm{kg}$ of ketamine for induction. Then, patients received an infusion of $100 \mathrm{ug} / \mathrm{kg} / \mathrm{min}$ propofol and $1 \mathrm{mg} / \mathrm{kg} / \mathrm{h}$ ketamine for maintenance.
After a minimum fasting period of 4 hours in infants and 6 hours in children, patients were admitted to the angiography unit; In the pre anesthetic holding area, an eutectic mixture of local anesthetic cream was applied to all patients' groins for femoral vascular access 60 minutes before the procedure.Then, all patients were premedicated with intramascular midazolam ( 0.07 to $0.08 \mathrm{mg} / \mathrm{kg}) \quad 30$ minutes before the procedure, an intravenous catheter $(20,22$ or 24 guage) was placed according to the age, and an infusion of D5 $0.3 \% \mathrm{NaCl}$ solution was started at a rate of 100 $\mathrm{mL} / \mathrm{kg} / 24$ hours.

\section{Monitored variables included;}

1- ECG monitoring,

2- Heart rate,

3- None-invasive blood pressure,

4- Pulse oximetry,

5- Respiratory rate,

6- Ramsey sedation scores (Ramsey et al, 1974).

- If awake: anxious, agitated, restless(1).

- cooperative,oriented,tranquil (2).

- responsive to command only (3).

- If asleep: - brisk response to light glabellar tap or loud auditory stimulus (4).

-sluggish response to light glabellar tap or loud auditory stimulus (5).

-no responseto light glabellar tap or loud auditory stimulus (6).

Values will be recorded as baseline, after induction, and every 15 minutes thereafter.

Intraoperatively, all patients breathed spontaneously. Supplemental 1.5 to 2 $\mathrm{L} / \mathrm{min}$ of $100 \%$ oxygen given via nasal cannula .Changes in systolic arterial pressure and heart rate recorded.

The sedation level of patients evaluated by Ramsey sedation score, when became less than level 5, additional doses of ketamine, $1 \mathrm{mg} / \mathrm{kg}$, administered in both groups. The anesthetic drug infusion discontinued 
SOHAG MEDICAL JOURNAL

Vol. 21 No.2 July 2017
Comparative study between Dexmedetomidine-Ketamine Hassan Ahmed M.et al when the groin bandage applied, and total drug doses recorded .

The side effects during the study recorded. The patients evaluated by Steward recovery scores, when became 6 , they transferred to the pediatric intensive care unit.

\section{ß Consciousness}

- Awake (3),

- Responds to verbal stimuli (2),

\section{Results}

- Responds to tactile stimuli (1),

- Not responding (0).

ß Airway

- Cough on command or cry (2),

- Maintains good airway (1),

- Requires airway assistance (0).

ß Motor

- Moves limbs purposefully (2),

- Non purposeful movement (1),

- Not moving (0) (Steward, 1975).

Table (1): Sedation score in both groups

\begin{tabular}{|l|l|l|l|}
\hline Time & DK (group) & PK (group) & P Value \\
\hline At base line & $2.64 \pm .492$ & $2.64 \pm .492$ & 1.000 \\
\hline After induction & $6.00 \pm .000$ & $6.00 \pm .000$ & N/A \\
\hline $15 \mathrm{~min}$ & $5.73 \pm .703$ & $6.00 \pm .000$ & 0.076 \\
\hline $30 \mathrm{~min}$ & $5.45 \pm .912$ & $5.50 \pm .889$ & 0.871 \\
\hline $45 \mathrm{~min}$ & & & \\
\hline $60 \mathrm{~min}$ & $5.80 \pm .632$ & $5.45 \pm .934$ & 0.339 \\
\hline $75 \mathrm{~min}$ & $6.00 \pm .000$ & $5.67 \pm .816$ & 0.447 \\
\hline $90 \mathrm{~min}$ & $4.00 \pm$ N/A & $5.00 \pm 1.414$ & 0.667 \\
\hline
\end{tabular}

This tables shows that the sedation score was similar in the two groups, with no-significant differences between the two groups except it recorded low levels at $15 \mathrm{~min}, 30 \mathrm{~min}$, and 75 min in DK group.

Table (2): Number of additional doses of ketamine in both groups

\begin{tabular}{|l|l|l|l|}
\hline Time & DK (group) & PK (group) & P Value \\
\hline At base line & $.00 \pm .000$ & $.00 \pm .000$ & N/A \\
\hline After induction & $.00 \pm .000$ & $.00 \pm .000$ & N/A \\
\hline $15 \mathrm{~min}$ & $2.27 \pm 2.27$ & $.00 \pm .000$ & .001 \\
\hline $30 \mathrm{~min}$ & $3.68 \pm 3.68$ & $.00 \pm .000$ & .001 \\
\hline $45 \mathrm{~min}$ & $.91 \pm .91$ & $.00 \pm .000$ & .001 \\
\hline $60 \mathrm{~min}$ & $.00 \pm .000$ & $.00 \pm .000$ & N/A \\
\hline $75 \mathrm{~min}$ & $.41 \pm 1.919$ & $.00 \pm .000$ & .001 \\
\hline $90 \mathrm{~min}$ & $.00 \pm .000$ & $.00 \pm .000$ & N/A \\
\hline
\end{tabular}

This table shows thatthenumber of additional doses of ketamine was significant between the two groups. because the need for boster doses of ketamine was more in DK group than PK group specially at $15 \mathrm{~min}, 30 \mathrm{~min}, 45 \mathrm{~min}$, and $75 \mathrm{~min}$. 
SOHAG MEDICAL JOURNAL

Vol. 21 No.2 July 2017
Comparative study between Dexmedetomidine-Ketamine Hassan Ahmed M.et al

Table (3): Recovery score in both groups

\begin{tabular}{|c|c|c|c|}
\hline Time & DK (group) & PK (group) & P Value \\
\hline At the end & $1.00 \pm .000$ & $5.41 \pm 20.680$ & 0.323 \\
\hline $5 \mathrm{~min}$ & $5.09 \pm 1.019$ & $8.32 \pm 18.274$ & 0.183 \\
\hline $10 \mathrm{~min}$ & $1.92 \pm .289$ & $5.50 \pm .730$ & 0.004 \\
\hline $15 \mathrm{~min}$ & $1.00 \pm$ N/A & $6.00 \pm .000$ & N/A \\
\hline
\end{tabular}

This table shows that, there was a significant difference between two groups, as the time for recovery was more rapid in DK (group) than in PK (group) the data is significant (.004) at 10 $\min$.

\section{Table (4): Side effects \& complications}

\begin{tabular}{|l|l|l|l|l|}
\hline & DK (group) & PK (group) & frequency \pm percent & P value \\
\hline Tachycardia & $.00 \pm .000$ & $.00 \pm .000$ & N/A & N/A \\
\hline Bradycardia & $.68 \pm .477$ & $.00 \pm .000$ & $15 \pm 34.1 \%$ & .035 \\
\hline Hypertention & $.00 \pm .000$ & $.00 \pm .000$ & N/A & N/A \\
\hline Hypotention & $.00 \pm .000$ & $.00 \pm .000$ & N/A & .001 \\
\hline Desaturation & $.09 \pm .104$ & $.50 \pm .512$ & $11 \pm 25 \%$ & .001 \\
\hline Vomiting & $.00 \pm .000$ & $.05 \pm .213$ & $1 \pm 2.3 \%$ & .001 \\
\hline Laryngospasm & $.32 \pm .477$ & $.14 \pm .351$ & $10 \pm 22.7 \%$ & N/A \\
\hline Agitation & $.00 \pm .000$ & $.00 \pm .000$ & N/A & .001 \\
\hline Shivering & $.00 \pm .000$ & $.14 \pm .351$ & $3 \pm 6.8 \%$ & N/A \\
\hline Hic cup & $.00 \pm .000$ & $.00 \pm .000$ & N/A & N/A \\
\hline Secretion & $.00 \pm .000$ & $.00 \pm .000$ & N/A & N/A \\
\hline Convulsions & $.00 \pm .000$ & $.00 \pm .000$ & & \\
\hline
\end{tabular}

This table shows the side effects\&complications that happened in both groups:- bradycardia was more common in DK (group) happened in (15) cases, desaturation was more common in PK (group) happened in (11) cases. laryngospasm happened in (10) cases in both groups, vomiting in (1) case, and shivering in (3) cases between the both groups .

\section{Discussion}

In this study, there is mild significant decrease on heart rate (HR decrease $<20 \%$ ) in DK group comparing with PK group.

Mason et al,2015. They reported that elevated doses of DEX (2-3 $\mu \mathrm{g} . \mathrm{kg}-1)$ over 10 minutes and infusion of 1.5-3.0 $\mu \mathrm{g} . \mathrm{kg}-1 . \mathrm{h}-1$ in children undergoing
MRI produce bradycardia in approximately $16 \%$ of those patients. Ayman et al,2014. reported that there are no significant differences in the hemodynamics (HR,SBP) between DK \& FK. In this study, there is significant decrease on oxygen saturation (SPO2) in PK group comparing with DK group. 
Ugley et al,2012. reported that The incidence of arterial desaturation was $26.7 \%(\mathrm{n}=8)$ in the KP group and $3.3 \%(\mathrm{n}=1)$ in the KPD group $(\mathrm{p}<0.05)$.

Tosun et al, 2006. Reported that no significant difference in (SPO2) arterial desaturation was $22.7 \%$ in $\mathrm{PK}$ group and $13.7 \%$ in DK group .

In this study, there is no significant difference the depth of sedation between two group $(\mathrm{P}>0.33)$, but the number of additional doses of ketamine (1 $\mathrm{mg} / \mathrm{kg}$ ) is more in DK group comparing with $\mathrm{PK}$ group $(\mathrm{P}=0.001)$.

Tosun et al, 2006. Reported that no significant difference in the depth of sedation between two group according to RSS \&BIS, In DK group, higher sedation scores were found at 30,45 , and 60 minutes than at baseline $(p<0.05)$; in PK group, sedation scores were higher at $15,30,45$, and 60 minutes. But the number of additional doses of ketamine $(1 \mathrm{mg} / \mathrm{kg})$ is more in DK group comparing with $\mathrm{PK}$ group $(\mathrm{P}<0.01)$.

Ugley et al,2012. reported that there is significant difference in the depth of sedation between two group according to RSS , as during local anesthesia infiltration, they observed movement in 27 patients in the KP group (all of them pulled their hands and feet) compared with 5 patients in the KPD group (all of them pulled their hands and feet) $(p<0.001)$. Also, the number of additional doses of propofol (0.2 $\mathrm{mg} / \mathrm{kg}$ ) is more in PK group comparing with DPK group $(\mathrm{P}<0.001)$.

In this study, we concluded that the recovery time is shorter in DK group (5-10 min) in compare with PK group (10-15 min) $(\mathrm{P}<0.004)$.

Ugley et al,2012. Reported that the recovery time is shorter in DPK group (3.13 min) in compare with PK group (5.86 min) $(\mathrm{P}<0.05)$.

Tosun et al,2006. Reported that the recovery time is longer in DK group
(46.54 min) in compare with PK group $(23.16 \mathrm{~min})(\mathrm{P}<0.05)$.

In this study, Bradycardia (HR decrease $<20 \%$ ) occurred in 15 cases $(34.1 \%)$ in DK group in compared with $\mathrm{PK}$ group (0\%) and $(\mathrm{P}=0.035)$.Desaturation $\quad$ (SPO2 decrease $>5 \%$ ) occurred in 11 cases $(25 \%)$ in PK group in compared with DK group 2 cases (4.5\%) and $(\mathrm{P}=0.001)$.Laryngospasm occurred in 7 cases $(15.9 \%)$ in DK group in compared with $\mathrm{PK}$ group 3 cases $(6.8 \%)$ and $\quad(\mathrm{P}=0.001)$.Shivering occurred in 3 cases $(6.8 \%)$ in PK group in compared with DK group (0\%) and $(\mathrm{P}=0.001)$. Vomiting occurred in 1 cases $(2.3 \%)$ in $\mathrm{PK}$ group in compared with DK group $(0 \%)$ and $(\mathrm{P}=0.001)$. None detected cases of tachycardia, hypertension, hypotension, increase oral secretion, convulsions, and hic cup in this study.

Tosun et al,2006. Reported that bradycardia (HR decrease $<20 \%$ ) occurred i cases $(18.2 \%)$ in DK group in compared with PK group 4cases (18.2\%) and tachycardia (HR increase $>20 \%$ ) occurred 2 cases $(9.1 \%)$ in DK group in compared with PK group 3 cases (13.6\%), and blood pressure (SBP increase $>20 \%$ ) occurred 2 cases $(9.1 \%)$ in DK group in compared with PK group 1 cases (4.5\%), and blood pressure (SBPdecrease $>20 \%$ ) occurred 3 cases $(13.6 \%)$ in DK group in compared with PK group 8 cases (36.4\%), and desaturation (SPO2 decrease $>5 \%$ ) occurred in 5 cases $(22.7 \%)$ in $\mathrm{PK}$ group in compared with DK group 3 cases (13.6\%), and laryngospasm occurred in 1 cases (4.5\%) in DK group in compared with $\mathrm{PK}$ group 1 cases $(4.5 \%)$, and shivering occurred in 1 cases $(4.5 \%)$ in PK group in compared with DK group $(0 \%)$,and agitation occurred in 1 cases $(4.5 \%)$ in DK group in compared with PK group (0\%), and hic cup occurred in 1 cases $(4.5 \%)$ in 
DK group in compared with PK group $(0 \%)$, and increase oral secretion occurred in $(0 \%)$ in DK group in compared with PK group 1 cases (4.5\%), but none detected cases of nausea and vomiting.

\section{Conclusion:}

Although all of the two anesthetic techniques were satisfactory, It was concluded that the use of dexamedetomidine in combination with Ketamine anesthesia is a safe, practical alternative for pediatric patients undergoing elective cardiac catheterization and may be preferable to ketamine/ propofol because of the significantly shorter recovery time, without hemodynamic or respiratory effects during the procedure despite being associated with decrease in HR (HR decrease $<20 \%$ ).

\section{References}

1. patients undergoing cardiac Akin A, Esmaoglu A, Guler G, et al: Propofol and Propofol-Ketamine in pediatric catheterization. Pediatric Cardiology (2005); 26:553-7.

2. Berman W, Fripp R, Rubler $M$ et al: Hemodynamic effects of ketamine in children undergoing cardiac catheterization. Pediatric Cardiology (1990), 11(2):72-76.

3. Bhana N, Goa KL, McClellan KJ: Dexmedetomidine. Drugs. (2000); 59:263-268.

4. Cravero JP, Blike GT, Beach M, et al: Incidence and nature of adverse events during pediatric sedation/anesthesia for procedures outside the operating room: Report from the Pediatric Sedation Research Consortium. Pediatrics (2006); 118:1087-96.

5. Ewen A, Archer DP, Samanani N, et al: Hyperalgesia during sedation: effects of barbiturates and propofol in the rat. Can J Anesth (1995); 42:532-40.

6. Hickey PR, Wessel DL, Streitz SL, et $a l$ :Transcatheter closure of atrial septal defects: hemodynamic complications and anesthetic management. Anesth Analg (1992);74:44-50.

7. Mullins CE, Malden, MA: Transseptal left heart catheterization. In: Mullins C, ed.Cardia Catheterization in Congenital Heart Disease: Pediatric andAdult : Blackwell Futura; (2006); 223-254.

8. Ramsay MA, Savege TM, et al: Controlled sedation with alphaxalonealphadolone. BMJ (1974); 22:656-659.

9. Steward DJ: A simplified scoring system for the post-operative recovery room. Can Anaesth Soc J (1975); 22:111-113.

10.Williams GD, Philip BM, Chu LF, et al: Ketamine does not increase pulmonary vascular resistance in children with pulmonary hypertension undergoing sevoflurane anesthesia and spontaneous ventilation. Anesth Analg (2007);105:1578-84.

11.Mason KP, Lonnqvist PA. : Bradycardia inperspective-not all reductions in heart rate need immediate intervention. Pediatr Anesth (2015); 25: 44-51.

12.A.Ulgey, R. Aksu, C. Bicer, et al : Is the Addition of Dexmedetomidine to a Ketamine-Propofol Combination in Pediatric Cardiac Catheterization Sedation Useful? Pediatr Cardiol (2012); 33:770-774.

13.Ayman A, Osama $M$, Mohamad $S$, et al : Comparative study between dexmedetomidineketamine and fentanyl-ketamine combinations for sedation in patients undergoing extracorporeal shock wave lithotripsy. A randomized double blinded study, Egyptian Journal of Anaesthesia (2014); 31, 35-41.

14.Tosun Z, Akm A, Gu 'ler G, Esmaog 7u $A$, et al : Dexmedetomidine-ketamine and propofol ketamine combination for anesthesia in spontaneously breathing pediatric patient undergoing cardiac catheterization. J Cardiothorac Vasc Anesth (2006) ; 20:515-519. 
SOHAG MEDICAL JOURNAL

Vol. 21 No.2 July 2017

109

PDF created with pdfFactory Pro trial version www.pdffactory.com 